\title{
Molecular Dynamics Simulations of Self-healing Topological Copolymers with Comb-like Structure
}

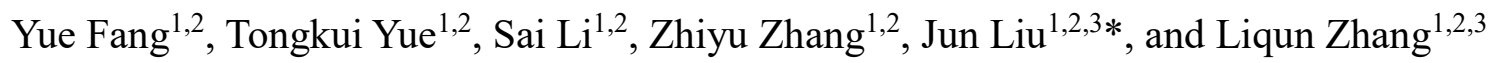

${ }^{1}$ Key Laboratory of Beijing City on Preparation and Processing of Novel Polymer Materials,

Beijing University of Chemical Technology, People's Republic of China

${ }^{2}$ Beijing Engineering Research Center of Advanced Elastomers, Beijing University of

Chemical Technology, People's Republic of China

${ }^{3}$ State Key Laboratory of Organic-Inorganic Composites, Beijing University of Chemical

Technology, People's Republic of China

\section{Setting of CG model and Interaction based on NCI analysis}

\subsection{From all-atom model to coarse-grained (CG) model}

The mapping from an all-atom model to a coarse-grained (CG) model (shown in Fig. S1) is based on two main principles: alternating copolymerization of MMA monomer and n-BA monomer as reported by Urban and convenience of structural parameter adjustment.

Firstly, Urban et al reported that self-healing behavior occurs in a narrow compositional range that are alternating, which is attributed to favorable interchain van der Waals forces. In our simulation, we set up a coarse-grained model for alternating copolymerization of two monomers. Monomer MMA is represented by beads of type D. Monomer n-BA consists of beads of three types (A, B, C), where the electron-absorbing ester group is set to type B and the distal methyl group on the branched chain is set to type C. Here, the diameter of type A, B, and $\mathrm{C}$ beads is $1 \sigma$, and the diameter of type $\mathrm{D}$ beads is variable. 
In addition, this coarse filtration model setting also facilitates the adjustment of structural parameters. First, we could adjust the distance between two neighboring branched chains by adjusting the diameter of the type D. Also, we could investigate the effect of relative molecular mass on molecular conformation by adjusting the mass of the type $\mathrm{D}$ on the backbone chain. Furthermore, we adjust the flexibility of the branched chains by adjusting the value of the coefficient $\mathrm{k}$ of the bond angle of monomer n-BA.
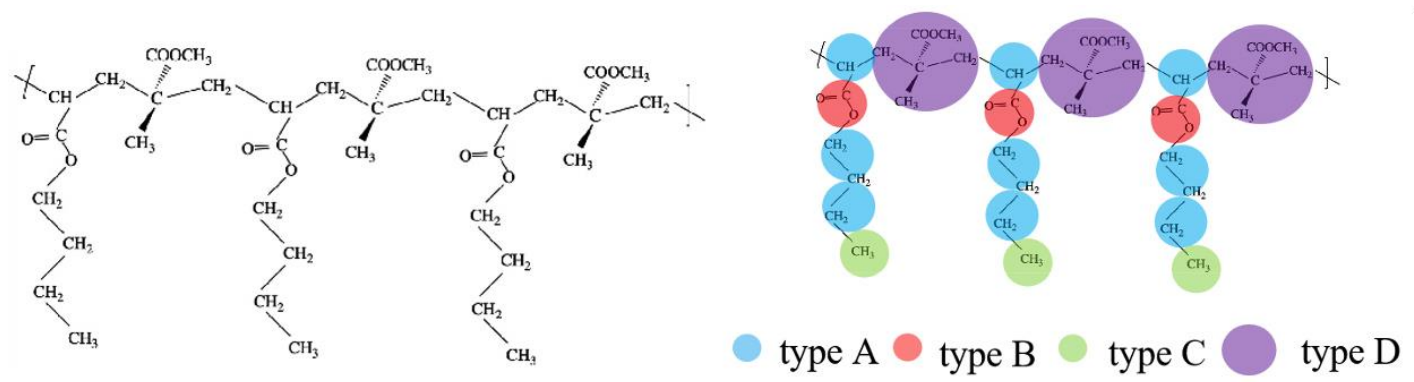

Figure S1. mapping from all-atomic model to CG model

\subsection{Interaction setting of CG model}

The results of NCI analysis showed that there are large steric repulsion (the red area) effects and mutual repulsive forces along the backbone chains of such comb-like molecules due to the presence of methyl methacrylate (MMA) and N-butyl acrylate (nBA) monomer. For example, as shown in Fig. S2, the red region corresponds to the space between methylene along the backbone chains as well as methyl of MMA and ester groups of nBA. Therefore, we set $r_{\text {cutoff }}$ between type A \& type D is $1.12 \sigma$, as well as type B \& type D, to model steric repulsion. And we also set the interaction to a large value, $10.0 \varepsilon$, to represent the strong steric repulsion. Also, the side chains between neighboring molecules exhibit more ordered arrangement due to weak interaction drives (the green area), so we model weak attraction interactions between type A \& type A, type A \& type B, type A \& type C by setting $r_{\text {cutoff }}$ equal $2.24 \sigma$. 

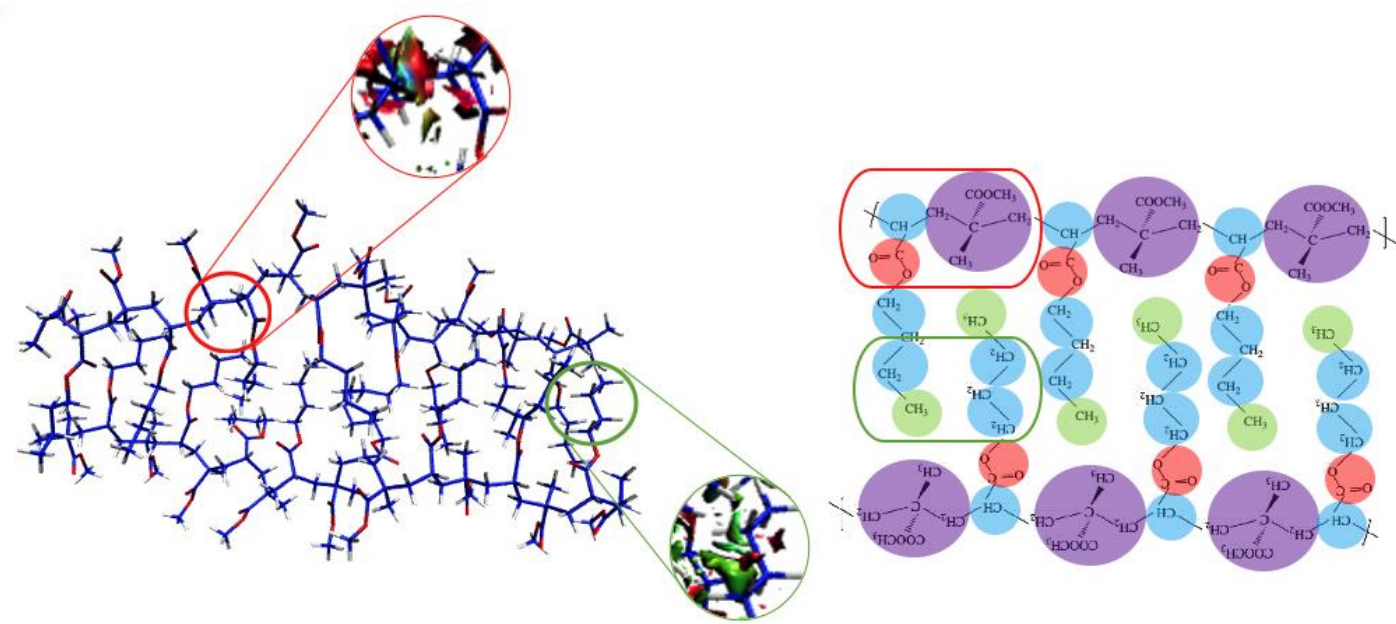

Figure S2. Schematics of correspondence of interaction parameters of CG model and all-atomic model

Urban's work illustrated that the side chains interactions were induced by the dipole interactions. ${ }^{1}$ Explicitly, the ester group of n-butyl acrylate displays negative electrical properties due to long-range electron absorption while the distal methyl group displays positive electrical properties. Therefore, we simulated this dipole-dipole interaction by setting the repulsive interaction between type $\mathrm{B} \&$ type $\mathrm{B}$, type $\mathrm{C} \&$ type $\mathrm{C}$ by setting $r_{\text {cutoff }}$ to $1.12 \sigma$. And the attractive interaction was set between type B \& type $\mathrm{C}$ by setting $r_{\text {cutoff }}$ to $2.50 \sigma$. And the comparison of parameters between the CG model and all-atomic model was listed in Table S1. More references about the setting of LJ interaction can be found in these references. ${ }^{2-}$ 
Table S1. Comparison of parameters between the CG model and all-atomistic model

\begin{tabular}{|cccc|cc|}
\multicolumn{2}{|c}{ CG model } & \multicolumn{2}{c|}{ all-atom model } \\
\hline \multicolumn{2}{|c|}{ Pair of beads } & $\varepsilon_{\mathrm{ij}} / \varepsilon$ & $\boldsymbol{r}_{\text {cutoff }}$ & NCI area & interactions \\
\hline type A & type D & 10 & 1.12 & red & steric repulsion \\
\hline type B & type D & 10 & 1.12 & red & steric repulsion \\
\hline type A & type A & 1.0 & 2.24 & green & van der Waals force \\
\hline type A & type B & 1.0 & 2.24 & green & van der Waals force \\
\hline type A & type C & 1.0 & 2.24 & green & van der Waals force \\
\hline type C & type D & 1.0 & 2.24 & green & van der Waals force \\
\hline type B & type B & 1.0 & 1.12 & - & induced dipoles \\
\hline type C & type C & 1.0 & 1.12 & - & induced dipoles \\
\hline type B & type C & 2.0 & 2.5 & - & induced dipoles \\
\hline
\end{tabular}

\section{Details of equilibrium process in MD simulation}

\subsection{Verification of equilibrium state}

Here, we verify equilibration state by observing the changes in the volume and internal energy of the system, and the system is considered to have reached equilibrium when the volume and internal energy of the system do not change with equilibrium time under NPT conditions. From the results in Fig. S3 (take system 2 for example), the non-bond energy and volume of the system remain basically unchanged after around 14 million steps of running (14000 $\tau$ ), and the system can be considered to have reached an equilibrium state. In the whole process of equilibrium, the temperature was fixed at $T^{*}=1.0$. The final number density is around 0.2 and mass density is around 0.9. Glass transition temperature $\left(T_{g}\right)$ around 0.56 and the polymers are in a melt (Fig. S4). 

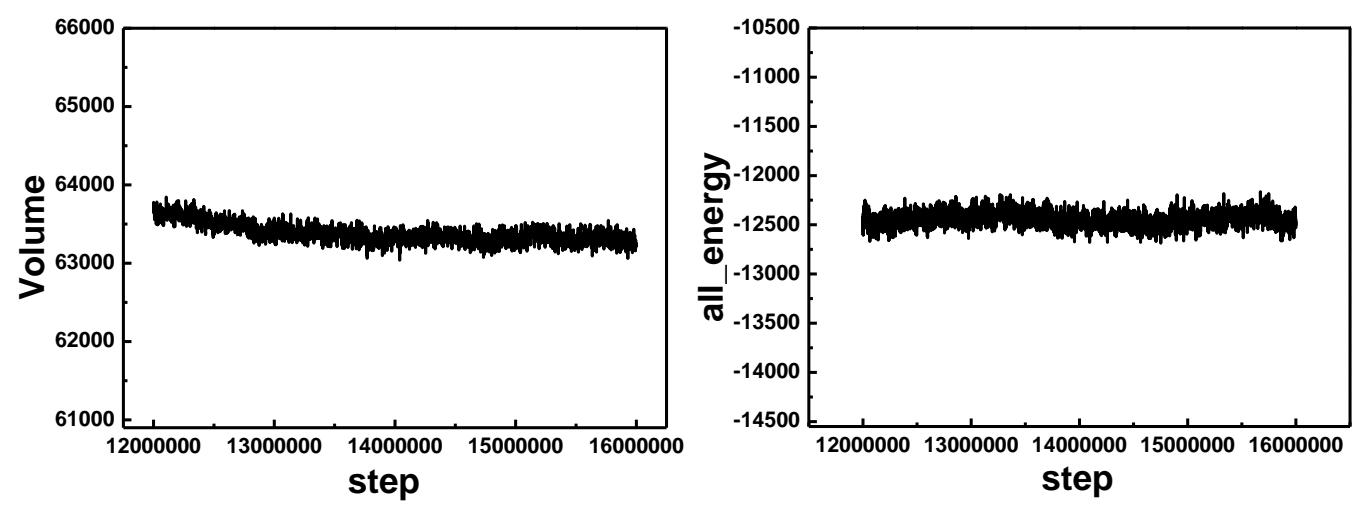

Figure S3. The volume and non-bond energy of system during the balance process

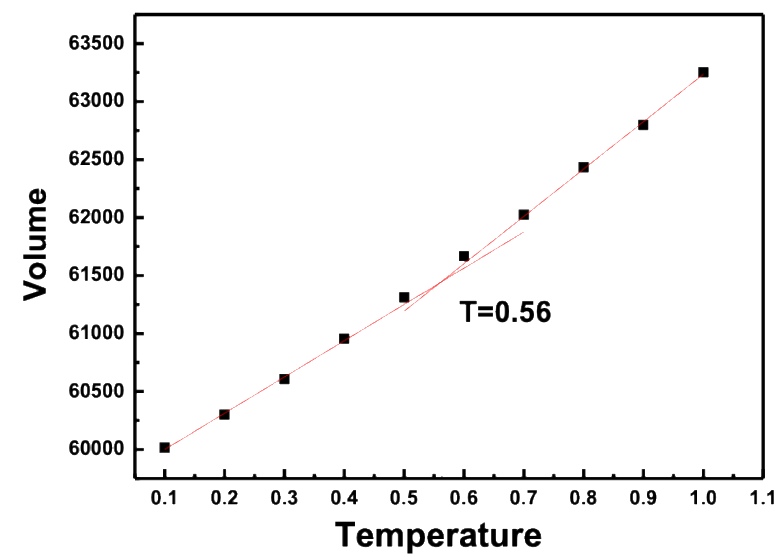

Figure S4. Glass transition temperature of system 2

\section{REFERENCES}

(1) Urban, M. W.; Davydovich, D.; Yang, Y.; Demir, T.; Zhang, Y.; Casabianca, L. Keyand-Lock Commodity Self-Healing Copolymers. Science 2018, 362 (6411), 220.

https://doi.org/10.1126/science.aat2975.

(2) Liu, J.; Gao, Y.; Cao, D.; Zhang, L.; Guo, Z. Nanoparticle Dispersion and Aggregation in Polymer Nanocomposites: Insights from Molecular Dynamics Simulation. Langmuir 2011, 27 (12), 7926-7933. https://doi.org/10.1021/la201073m.

(3) Shen, J.; Li, X.; Shen, X.; Liu, J. Insight into the Dispersion Mechanism of PolymerGrafted Nanorods in Polymer Nanocomposites: A Molecular Dynamics Simulation Study. Macromolecules 2017, 50 (2), 687-699. https://doi.org/10.1021/acs.macromol.6b02284.

(4) Chen, Y.; Xu, Q.; Jin, Y.; Qian, X.; Liu, L.; Liu, J.; Ganesan, V. Design of End-to-End Assembly of Side-Grafted Nanorods in a Homopolymer Matrix. Macromolecules 2018, 51 (11), 4143-4157. https://doi.org/10.1021/acs.macromol.8b00292. 